Meta

Journal des traducteurs

Translators' Journal

\title{
Colloque de traduction en Angleterre
}

\section{Robert Larose}

Volume 28, numéro 4, décembre 1983

URI : https://id.erudit.org/iderudit/003412ar

DOI : https://doi.org/10.7202/003412ar

Aller au sommaire du numéro

Éditeur(s)

Les Presses de l'Université de Montréal

ISSN

0026-0452 (imprimé)

1492-1421 (numérique)

Découvrir la revue

Citer cette note

Larose, R. (1983). Colloque de traduction en Angleterre. Meta, 28(4), 432-433.

https://doi.org/10.7202/003412ar d'utilisation que vous pouvez consulter en ligne.

https://apropos.erudit.org/fr/usagers/politique-dutilisation/ 


\section{COLLOQUE DE TRADUCTION EN ANGLETERRE}

Les $16,17,18$ et 19 juin 1983 s'est tenu à Londres un colloque franco-britannique sur la traduction organisé par l'UER Angellier de l'Université de Lille III et l'Institut français du Royaume-Uni.

Les quelque 27 communications devaient porter sur l'un ou l'autre des trois thèmes du colloque, soit 1. La traduction comme phénomène social et culturel, 2 . Les problèmes actuels des approches linguistiques et didactiques en traduction et 3 . Traduction et herméneutique. L'aspect didactique, malheureusement, n'a nullement été abordé. Mais cela n'est pas tout à fait étonnant étant donné que la traduction en Europe n'est pas tant l'objet d'un enseignement universitaire sui generis qu'un moyen d'évaluer les compétences langagières d'un candidat inscrit dans un programme de langue (donc absence de dictionnaires, absence de notes accompagnant les textes à traduire, terrorisme du corrigé, etc., pratiques inconciliables avec les règles fondamentales d'enseignement de la traduction). En revanche, il n'est aucunement surprenant de constater que le quart des conférences a porté sur divers aspects de la linguistique différentielle. Il est important de souligner à ce titre qu'au contraire des recherches menées par certains comparatistes canadiens (cf. Vinay et Darbelnet), les études différentielles poursuivies actuellement en France ajoutent à la rigueur de leurs analyses une linguistique de l'énonciation, éliminant ainsi le principal reproche que les traductologues formulent à l'égard des comparatistes, c'est-à-dire celui consistant à ne pas paroliser les actes de langage. Àcet égard, les travaux de J. Guillemin-Flescher, Syntaxe comparée du français et de l'anglais - Problèmes de traduction et de G. Garnier, Éléments de systématique verbale comparée du français et de l'anglais. Contribution à une théorie de la traduction devront maintenant figurer parmi les lectures obligatoires de tout cours de linguistique différentielle de niveau universitaire.

Encore une fois, le débat principal tout au long du colloque a porté sur la question de la fidélité (au signifiant-signifié de la langue de départ). Le transfert du signifiant en particulier a surtout retenu l'attention des participants, car la moitié des conférences portaient sur des aspects divers de la traduction littéraire : tonalité, rythme, résonance, prosodie, etc. L'autre quart était consacré entre autres au rapport entre psychanalyse et traduction (Rozenberg, Sibony) et à la traduction philosophique (grâce à l'inattendue et éclairante présence de J.-R. Ladmiral). Il est malheureux d'ailleurs que $\mathbf{H}$. Meschonnic ait dû se désister puisque les participants auraient peut-être pu assister in vivo à une reprise du débat amorcé par ces deux derniers chercheurs dans le numéro spécial sur la traduction de la revue Langue française ( ${ }^{\circ} 51$, septembre 1981).

Impressionnants par le contenu culturel de leurs communications et par l'éloquence avec laquelle ils transmettent leurs idées, les conférenciers ont su apporter de nouveaux éclairages sur nombre de problèmes relatifs à la forme des textes et au rôle d'éléments linguistiques supra-segmentaux en traduction (résonance (Vreck), prosodie (Gobard), rythme (Robel)). Ils ont particulièrement réussi à élargir les perspectives de recherches universitaires à caractère multidisciplinaire, parmi lesquelles la traduction de textes anciens (Charpin, Buridant, Kelly), le rapport entre traduction et philosophie (Ladmiral, Newmark) et celui entre traduction et psychanalyse. La réflexion sur les vecteurs d'historicité et de prestige des textes à traduire, ainsi que sur le caractère parfois sacré de leur forme, doit se poursuivre si l'on espère qu'un jour quelqu'un puisse proposer une première théorie de la traduction.

ROBERT LAROSE 